\title{
Simultaneous extraction and purification of fucoxanthin from Tisochrysis lutea
} microalgae using compressed fluids

Rocío Gallego ${ }^{1}$, Charles Tardif ${ }^{1}$, Celina Parreira², Tiago Guerra², Maria João Alves², Elena Ibáñez ${ }^{1}$, Miguel Herrero ${ }^{1 *}$

${ }^{1}$ Laboratory of Foodomics, Institute of Food Science Research (CIAL, CSIC-UAM), Calle Nicolás Cabrera 9, 28049 - Madrid, Spain.

${ }^{2}$ A4F - Algae for Future, Campus do Lumiar, Estrada do Paço do Lumiar, Edif. E, R/C, 1649-038 Lisboa, Portugal

* Corresponding author: Dr. Miguel Herrero, Laboratory of Foodomics, Institute of Food Science Research (CIAL, CSIC-UAM), Calle Nicolás Cabrera 9, 28049 - Madrid, Spain. Email: m.herrero@csic.es; Tel.: +34-910-017-946

Running title: Simultaneous Extraction and Purification of Fucoxanthin from Tisochrysis lutea

Abbreviations: AC, Activated charcoal; BHT, Butylated hydroxytoluene; CE, Conventional extraction; DAD, Diode array detection; DW, Dry weight; EtAc, Ethyl acetate; EtOH, Ethanol; GRAS, Generally recognized as safe; LED, Light-emitting diode; LHC, Light harvesting complex; MTBE, Methyl tert-butyl ether; NL, Natural light; PLE, Pressurized liquid extraction; RP: Reversed-phase; RSM, Response surface methodology; SFDW: Salt-free dry weight.

Keywords: activated charcoal, adsorbents, fucoxanthin, purification, Tisochrysis lutea 
The marine microalga Tisochrysis lutea, a Haptophyta with a thin cell wall and currently used mainly in aquaculture is a potential source of several bioactive compounds of interest such as carotenoids. In the present study, the simultaneous extraction and purification of fucoxanthin, the main carotenoid from $T$. lutea, was optimized using pressurized fluid extraction followed by in-cell purification. An experimental design was employed to maximize carotenoids' extraction; the experimental factors chosen were: (i) percentage of ethanol/ethyl acetate $(0-100 \%)$, (ii) temperature $\left(40-150{ }^{\circ} \mathrm{C}\right)$ and (iii) number of static extraction cycles $(1-3)$. The maximum carotenoids' recovery, mainly fucoxanthin, was obtained with pure ethyl acetate at $40^{\circ} \mathrm{C}$ using one extraction cycle, achieving values of $132.8 \mathrm{mg}$ of carotenoids per $\mathrm{g}$ of extract. Once the optimum extraction conditions were confirmed, in-cell purification strategies using different adsorbents were developed to obtain fucoxanthin-enriched extracts. Activated charcoal showed potential retention of chlorophylls allowing an effective purification of fucoxanthin in the obtained extracts. Chemical characterization of extracts was carried out by reversed-phase high-performance liquid chromatography with diode array detection. Therefore, a selective fractionation of high value compounds was achieved using the proposed green downstream platform based on the use of compressed fluids. 


\section{INTRODUCTION}

Nowadays, there is an enormous interest in discovering new sources of natural bioactive compounds with benefits for human health. In this regard, microalgae have gained importance as promising sources of bioactives due to the diversity of compounds that can be extracted from different strains and their high photosynthetic efficiency and high growth rate, among many other properties [1]. It is well-known that under controlled conditions, mostly adverse or stress conditions, some microalgae can over-produce particular bioactive compounds. The most common examples are the overproduction of $\beta$-carotene by Dunaliella salina and astaxanthin by Haematococcus pluvialis, but new species are now being explored [2]. For instance, Mulders et al. [3] found a negative correlation between nutrient depletion and growth of the haptophyte Isochrysis aff. galbana (clone T-iso) although, in contrast, an accumulation of some bioactives occurred, specifically, carotenoids such as 3-hydroxyechinenone, echinenone, and diatoxanthin. This marine microalga, also known as Tisochrysis lutea, belongs to the Isochrysidaceae family of non-calcifying organisms and has been widely used for feed in aquaculture [4]. However, considering its chemical composition and content on interesting compounds such as carotenoids and polyunsaturated fatty acids, this strain could be pointed out as a promising source of bioactive compounds for other purposes, including cosmetic, pharmaceutical, food and nutraceutical industries. In this sense, a recent study demonstrated that a $T$. lutea-rich diet in rats was well tolerated and helped to control dyslipidemias although its salt content might pose a safety issue in humans $[5]$.

As already mentioned, carotenoids are among the most relevant bioactive compounds in T. lutea. These lipophilic compounds are related to many physiological functions, 
including protection against oxidation and excess of light, or even controlling the microalgal growth [6]. Moreover, these compounds have been demonstrated to play an important role in reducing or preventing many human diseases, and also enhancing immune responses against several infections [7]. Among carotenoids, fucoxanthin is one of the most studied and promising compounds. It has an unusual allenic bond (see Figure S1), which seems to be responsible for its high antioxidant activity, but this carotenoid has also been related to other biological functions including cardioprotective, neuroprotective, anti-cancer, anti-obesity, anti-inflammatory, anti-diabetic and antiangiogenic activities, among others [1]. This pigment, that can be found mainly in brown macroalgae [8], is the main carotenoid of Tisochrysis lutea.

The development of advanced environmentally friendly extraction techniques and processes to recover carotenoids, such as fucoxanthin, from natural sources is a current challenge [9]. The advantages of using compressed fluid-based extraction techniques to achieve carotenoid-enriched extracts are more than justified, not only due to the reduction of toxic solvents but also due to the increase of extraction efficiency. One of these methods is pressurized liquid extraction (PLE), which is characterized by the use of high temperatures and pressures enough to maintain the solvent in the liquid state. PLE employs relatively small volumes of solvents in comparison with conventional extractions [10]. Under these conditions, extractions are faster due to the increase of mass transfer rate and the deeper contact between the solvent and the matrix, which also leads to an increase of the extraction yield. To comply with the green chemistry principles, generally recognized as safe (GRAS) solvents such as ethanol, water or ethyl lactate are mainly used for these purposes, and many studies have demonstrated their application in the extraction of carotenoids [10]. 
Nevertheless, PLE may not offer a high selectivity towards those compounds, and even optimization of extraction parameters could not be enough to obtain extracts selectively enriched in the target compound. For this reason, the use of simultaneous purification procedures is of interest. In this sense, the use of adsorbents as a purification step is currently being considered as a feasible green and cheap alternative. Thus, adsorbents can selectively recover the compounds of interest, implying a later desorption step, but also they can be used to remove the unwanted components from a natural matrix in other analytical fields, such as pesticide residues and other environmental contaminants [11-13]. For instance, Osman et al [14] developed a platform in which PLE and the use of sorbents were integrated to selectively extract several contaminants from soils. As expected, the efficiency and selectivity of the process depended on the properties of each sorbent, organic compounds of interest and all operating parameters of the PLE. The main issue regarding the extraction of carotenoids from microalgae is that due to their polarity other non-desired pigments (such as chlorophylls) are co-extracted. Up to now the removal of these pigments has been achieved with complex methods such as acid treatments [15] or saponification after extraction [16] that are far from being environmentally respectful.

Therefore, in this study, the main objective was to develop an in-line purification process simultaneously combining the use of PLE and adsorbents to efficiently obtain fucoxanthin-enriched extracts from $T$. lutea microalga.

\section{MATERIALS AND METHODS}

\subsection{Samples and reagents}


HPLC-grade methyl tert-butyl ether (MTBE), methanol, ethyl acetate (EtAc), acetone and ethanol (EtOH) were acquired from VWR (Leuven, Belgium). Butylated hydroxytoluene (BHT) and fucoxanthin, $\beta$-carotene and chlorophyll $a$ were purchased from SigmaAldrich (St Louis, MO, USA). Sea sand (0.25-0.30 mm particle diameter) was obtained from Panreac (Castellar del Vallés, Spain). Ultrapure water used was acquired from a Milli-Q system (Millipore, Billerica, MA, USA). Regarding the adsorbents, aluminum oxide 150 basic (Type T), activated charcoal powder and molecular sieve adsorbent (13X, 100-120 mesh) were acquired from Sigma-Aldrich, whereas ISOLUTE C18 and ISOLUTE ENV+ were purchased from Biotage (Grenzach-Wyhlen, Germany).

Tisochrysis lutea microalga was kindly supplied by A4F - Algae for Future (Lisbon, Portugal). T. lutea (CCAP 927/14) was obtained from the Culture Collection of Algae and Protozoa at the Scottish Association for Marine Science (Oban, UK). Cultures were grown from August to October of 2018 in A4F's Pilot unit in Lisbon using a flat panel photobioreactor (FP-PBR) technology outdoors, in the East - West orientation. The reactors were $9 \mathrm{~cm}$ in depth and contained $270 \mathrm{~L}$ of $A 4 \mathrm{~F}^{\prime} \mathrm{s}$ industrial medium as cultivation medium with strict $\mathrm{pH}$ between 7 and 8 and temperature controlled bellow $28^{\circ} \mathrm{C}$. A continuous stirring was applied through aeration (air supplemented with $0.5 \%$ $\mathrm{CO}_{2}$ ). Two cultivations started side by side with the same optical density (600 nm) of 0.55. Besides natural light (NL) which illuminated both reactors, one of the systems was impinging an average light intensity of $161 \pm 52 \mu \mathrm{mol} \mathrm{m}^{-2} \mathrm{~s}^{-1}$ on each side of the reactor. Both cultivations were maintained for 65 days under a semi-continuous regime. The concentration of microalgal biomass was measured as g salt-free dry weigh (SFDW) per 
L. Biomass was harvested by centrifugation, frozen at $-20^{\circ} \mathrm{C}$, lyophilized and used for further fractionation.

\subsection{Extraction methods}

142 In order to choose the optimum batch (NL or LED) and also the main extraction 143 parameters to recover carotenoids, a screening was performed employing different extraction methods (conventional or pressurized extraction), different solvents (acetone or ethyl acetate) and temperatures (40 and $\left.100{ }^{\circ} \mathrm{C}\right)$. When these parameters were selected, an optimization of PLE procedure was performed following an experimental design as described in Section 2.3. The selection of the optimum conditions were done based on the following variables: extraction yield, expressed as percentage (g of extract $_{D W} / \mathrm{g}$ initial biomass $\mathrm{DW} \times 100$ ); total carotenoids and chlorophylls, shown as $\mathrm{mg}$ carotenoids or chlorophylls per g of extractow, and; ratio between carotenoids and chlorophylls (mg carotenoids / mg chlorophylls per g of extract ${ }_{D w}$ ).

\subsubsection{Conventional extraction}

Conventional extractions (CE) were performed (in duplicate) to determine the total extractable carotenoids and chlorophylls in T. lutea, following the method described by Gilbert-López et al. [17], with some modifications. Briefly, 200 mg of T. lutea lyophilized biomass was mixed with $20 \mathrm{~mL}$ acetone or ethyl acetate containing $0.1 \%(\mathrm{w} / \mathrm{v})$ BHT. Then, the mixture was maintained under agitation at $250 \mathrm{rpm}$ for $24 \mathrm{~h}$ protected from light. After centrifugation (10,000 rpm at $4{ }^{\circ} \mathrm{C}$ for $\left.10 \mathrm{~min}\right)$, the supernatant was collected, transferred to a new glass vial and the solvent was dried using a nitrogen stream. Dry extracts were weighted and stored at $-20^{\circ} \mathrm{C}$ until further analysis.

\subsubsection{Pressurized liquid extraction}


(40 or $100{ }^{\circ} \mathrm{C}$ ) using ethyl acetate as solvent and one static extraction cycle.

Subsequently, for the PLE optimization, extractions were carried out at different extraction temperatures ( 40 to $120^{\circ} \mathrm{C}$ ), solvent compositions (percentage of ethanol in ethyl acetate) and number of static extraction cycles (1 to 3 cycles), according to the experimental design described in the section 2.3 (see Table S1). Before each extraction, an extraction cell heat-up step was performed for a given time that is fixed by the equipment (i.e., 5 min when the extraction temperature was set at $40^{\circ} \mathrm{C}$, and 6 min at $120^{\circ} \mathrm{C}$ ). All extractions were done using $11 \mathrm{~mL}$ extraction cells at $10.5 \mathrm{MPa}$ and $20 \mathrm{~min}$ as total static extraction time independently of the number of cycles performed. The extraction cell was filled with $1 \mathrm{~g}$ of algae sandwiched between two layers of sea sand $(2 \mathrm{~g})$ as can be observed in Figure 1A. The total volume of solvent amount used in each extraction varied from 18 to $20 \mathrm{~mL}$. All experiments were performed at least in duplicate. Extracts were dried using a stream of nitrogen, protected from light and stored under refrigeration $\left(-20^{\circ} \mathrm{C}\right)$ until further analysis.

\subsection{Pressurized liquid extraction: experimental design}

In order to optimize the extraction of carotenoids using PLE, an experimental design was proposed. A 3-level factorial experimental design $3^{3}$ (including three center points) was selected based on three factors: percentage of ethanol in ethyl acetate (0 to $100 \%$ $\mathrm{EtOH}$ ), temperature $\left(40\right.$ to $120^{\circ} \mathrm{C}$ ) and number of static extraction cycles ( 1 to 3 ) for a total of 30 experimental runs (see Table S1), since experiments were done in duplicate. 
performed using response surface methodology (RSM) using Statgraphics Centurion XVI software (StatPoint Technologies, Inc., Warrenton, VA, USA). The influence of each experimental factor on the response variables was studied at a $95 \%$ of level of confidence. In order to evaluate the effect of each factor for each of the response variables, the standardized Pareto charts and their respective response surfaces were analyzed, accepting the significances at $p \leq 0.05$. extraction

Five different adsorbents (isolute $\mathrm{C} 18$, isolute $\mathrm{ENV+}$, aluminum oxide, molecular sieve and activated charcoal) were studied to produce a simultaneous purification of the fucoxanthin obtained during the PLE extraction. Therefore, the same process conditions obtained as optimum extraction conditions in the experimental design were used. The experiments were proposed following a method described by Osman et al. [14], with some modifications. Briefly, $1.0 \mathrm{~g}$ of adsorbent was added at the bottom of the extraction cell and then sand and algal biomass were added as described above (Figure 1B). After selecting the most suitable adsorbent, different amounts of adsorbent $(0.25$ stored in dark under at $-20^{\circ} \mathrm{C}$ until analysis. All experiments were performed at least in duplicate.

\subsection{Chemical characterization of Tisochrysis lutea extracts}

\subsubsection{Total carotenoids and total chlorophylls determination}

A simple spectrophotometric method to determine total carotenoids and total chlorophylls contents in the extracts was used, based on their characteristic absorbance, 
as described elsewhere [18]. The extracts were dissolved in methanol at a concentration of $0.05 \mathrm{mg}$ per $\mathrm{mL}$ and the absorbance was recorded at 470 and $665 \mathrm{~nm}$ for carotenoids and chlorophylls, respectively. A calibration curve using fucoxanthin $(0.63-20.00 \mu \mathrm{g} / \mathrm{mL})$ as an external standard was employed to estimate total carotenoids content

214 (considering fucoxanthin the main carotenoid present in T. lutea), whereas chlorophyll $a(0.63-20.00 \mu \mathrm{L} / \mathrm{mL})$ was used as an external standard to determine total chlorophylls content. Results were expressed as mg carotenoids or chlorophylls per g extract

2.5.2. Identification of carotenoids and chlorophylls by liquid chromatography coupled

\section{to diode array detection}

The identification of pigments contained on the different extracts was performed by HPLC coupled to diode array detection and mass spectrophotometry (HPLC-DAD-MS), according to a method described by Gallego et al. [19], with some modifications. The equipment used was an Agilent 1100 series liquid chromatograph (Santa Clara, CA, USA) coupled to a diode-array detector (DAD). A YMC-C30 reversed-phase column $(250 \times 4.6$ $\mathrm{mm}, 5 \mu \mathrm{m}$ particle size; YMC Europe, Schermbeck, Germany) and a pre-column YMCC30 (10 $\times 4 \mathrm{~mm}, 5 \mu \mathrm{m}$ particle size) were used for the analysis. The following mobile phases were employed at a flow rate of $0.8 \mathrm{~mL}$ per min: methanol-MTBE-water (90:7:3, $\mathrm{v} / \mathrm{v} / \mathrm{v})$ as solvent $A$ and methanol-MTBE $(10: 90, \mathrm{v} / \mathrm{v})$ as solvent $B$. The linear step-wise gradient employed for elution was: $0 \mathrm{~min}, 5 \% \mathrm{~B} ; 7 \mathrm{~min}, 10.5 \% \mathrm{~B} ; 12 \mathrm{~min}, 15 \% \mathrm{~B} ; 17 \mathrm{~min}$, $30 \% \mathrm{~B} ; 19 \mathrm{~min}, 40 \% \mathrm{~B} ; 24 \mathrm{~min}, 100 \% \mathrm{~B} ; 30 \mathrm{~min}, 5 \% \mathrm{~B}$. The injection volume was $10 \mu \mathrm{L}$. The diode array detector (DAD) recorded at 280, 450 and $660 \mathrm{~nm}$, although spectra from 240 to $770 \mathrm{~nm}$ were also obtained (peak width $0.1 \mathrm{~min}(2 \mathrm{~s})$, slit $4 \mathrm{~nm}$ ). The instrument was controlled by LC ChemStation 3D Software Rev. B.04.03 (Agilent Technologies, Santa Clara, CA, USA). All extracts were dissolved in pure ethanol at an appropriate 
concentration (1-10 mg/mL) and filtered using $0.45 \mu \mathrm{m}$ nylon filters prior to analysis.

For peak identification, external standards of fucoxanthin and chlorophyll $a$ were injected, and UV spectra and MS fragmentation were also analyzed. The HPLC-DAD instrument was directly coupled to an ion trap mass spectrometer (Agilent ion trap 6320, Agilent Technologies, Santa Clara, CA, USA) via an atmospheric pressure chemical ionization (APCI) interface. All analyses were performed under positive ionization mode using the following parameters: drying temperature, $350^{\circ} \mathrm{C}$; vaporizer temperature, 400 ${ }^{\circ} \mathrm{C}$; drying gas flow rate, $5 \mathrm{~L} \mathrm{~min}{ }^{-1}$; capillary voltage, $-3.5 \mathrm{kV}$; nebulizer gas pressure, 60 psi; corona current (which sets the discharge amperage for the APCI source), $4000 \mathrm{nA}$. Full scan spectrum was obtained in the range from $\mathrm{m} / \mathrm{z} 150$ to 1200 . Automatic tandem mass spectrometry (MS/MS) analyses were also carried out, fragmenting the two highest precursor ions (10,000 counts threshold; 1 V Fragmentor amplitude).

\section{RESULTS AND DISCUSSION}

3.1 Influence of growing conditions on fucoxanthin content in Tisochrysis lutea biomass In this work, two batches of $T$. lutea biomass grown under different illumination conditions were compared to study how light intensity may influence fucoxanthin accumulation. It is known that fucoxanthin plays an important role during photosynthesis since it is present in light-harvesting complexes (LHCs); thus, differences on light intensity during production could determine the amount of this carotenoid within the biomass [20]. Indeed, it has been confirmed that different levels of 256 fucoxanthin could be achieved by applying different LED light colors in other algae [21], confirming the hypothesis of differences in fucoxanthin content. The use of artificial light 
258 (LEDs) on both sides of the reactor increased $T$. lutea volumetric biomass productivity in

259

260

261

262

263

264

265

266

267

268

269

270

271

272

273

274

275

276

277

$62 \%$ (Figure 2). Volumetric biomass productivities of $0.03 \pm 0.01$ and $0.05 \pm 0.02 \mathrm{~g} / \mathrm{L} / \mathrm{d}$ were attained for cultures in the absence or in the presence of LEDs, respectively. Accordingly, it was possible to achieve a maximum volumetric biomass concentration of 1.55 and 2.24 gSFDW $\mathrm{L}^{-1}$. To select the growing conditions producing a higher accumulation of carotenoids, mainly fucoxanthin, different extraction conditions were tested. Simultaneously, the influence of the extraction solvent, extraction pressure and temperature were also studied.

\subsubsection{Effect of solvent on extraction yield and carotenoid content}

A conventional extraction process was first applied to select the solvent providing a higher recovery of carotenoids (fucoxanthin) from the two T. lutea biomasses. Acetone is the most widely used solvent for the extraction of carotenoids, as described in many studies $[17,18,22-24]$, and therefore, was selected as a benchmark solvent. Nevertheless, based on the chemical nature of fucoxanthin, ethyl acetate is proposed in this work as a more environmentally respectful option. As can be observed in Figure S2A, there were no significant differences in terms of total extraction yield between the two batches (LED and NL) independently of the solvent employed, meaning that both behave similarly. However, the sample grown under LED supplementation seemed to produce a higher total extraction yield. In contrast, the content in carotenoids (Figure S2B) was significantly higher in the NL batch regardless of the solvent used, being this difference more marked when the extraction occurred in ethyl acetate. Based on these results, ethyl acetate was selected as possible GRAS extraction solvent which is in line with other previously published reports dealing with the extraction of fucoxanthin from other different microalgae species [25]. 


\subsubsection{Effect of pressure on extraction yield and carotenoid content}

283

284

285

286

287

Pressure is one of the most relevant parameters that influences the extraction of compounds, since it helps to penetrate within the sample and, thus, to increase the contact between the solvent and the sample. Moreover, the use of PLE usually provides higher extraction yields and efficiency compared to conventional extraction techniques. Once the extraction solvent was selected, a PLE procedure was compared to the conventional extraction process using similar extraction conditions $\left(40^{\circ} \mathrm{C}\right)$. Figure $\mathrm{S} 2 \mathrm{C}$ and S2D summarize the results found. Whereas the extraction yield (Figure S2C) seemed to be higher in the LED batch using conventional extraction, the carotenoid content (Figure S2D) was significantly higher for both batches using PLE. Considering that this is the most relevant parameter to be maximized, the use of PLE was regarded as more suitable.

Although the effect of pressure during PLE has repeatedly shown as negligible once this parameter is high enough to maintain the solvent in the liquid state, compared to conventional room conditions, the use of high pressures allows an increase on mass transfer rates thanks to a better penetration of the solvent into the sample particles, reaching deeper areas and increasing the surface contact [10]. Besides, theoretically, the pressure may also exert a weakening effect on the matrix structure, thus, further favoring the extraction of its components.

\subsubsection{Effect of temperature on extraction yield and carotenoid content}

To finish the initial comparison between the two $T$. lutea batches and to confirm the possibility to recover higher fucoxanthin amounts from the biomass grown under natural light, different extraction temperatures $\left(40\right.$ and $\left.100^{\circ} \mathrm{C}\right)$ were studied using PLE. These two extraction temperature conditions are different enough to evaluate the 
influence of this parameter on the extraction of fucoxanthin, as we have previously shown [25]. Extraction temperature is one of the most influential parameters during pressurized extractions since the solvent may have its physico-chemical properties modified as a result of the use of high temperatures while the liquid state is still maintained. To carry out these experiments, and according to the previous results, the extractions were performed using pressurized ethyl acetate extraction for $20 \mathrm{~min}$. As expected, extraction yield was increased with temperature (Figure S2E), since the use of a high temperature fosters an increase in solubility and mass transfer rate and, thus, higher recoveries. In the case of carotenoids, as can be observed in Figure S2F, no statistically significant differences were observed for the same batch between the two tested temperatures. In general, carotenoids may be negatively affected by high temperatures which are often correlated with an important decrease of carotenoids content. Nevertheless, some studies have revealed the importance of analyzing this parameter case-by-case, particularly, when PLE processes are used, considering the particular extraction conditions that are applied. For instance, Gallego et al. [19] showed that carotenoids content recovered from Porphyridium cruentum microalgae was higher extracting at $125^{\circ} \mathrm{C}$ compared to $50{ }^{\circ} \mathrm{C}$ using PLE.

Analyzing all the results obtained, NL batch was confirmed as being richer in carotenoids (fucoxanthin) compared to the batch produced under LED supplementation. Other authors have also demonstrated that lower light intensities were more favorable to produce $P$. tricornutum biomass with higher carotenoid content [26]. Therefore, NL $T$. lutea biomass was selected to proceed with the exhaustive optimization of the extraction conditions to maximize the recovery of carotenoids (fucoxanthin) using PLE. 
Based on the mentioned preliminary results, an experimental design was applied to study the influence of some parameters involved in the extraction of carotenoids (fucoxanthin) from T. lutea. The factors studied were extraction temperature (40 - 120 $\left.{ }^{\circ} \mathrm{C}\right)$, the number of static extraction cycles ( 1 - 3 cycles) maintaining a total static extraction time of $20 \mathrm{~min}$, and solvent composition. Although preliminary results showed that ethyl acetate was the most suitable solvent for fucoxanthin extraction, considering that ethanol has been also previously employed for the extraction of carotenoids from different microalgae $[25,27,28]$, the influence of the content of ethanol in the solvent mixture was evaluated. The use of ethanol was aimed to further increase the sustainability of the process considering that it is widely considered as GRAS. Three response variables were considered: extraction yield, total carotenoids content, and the ratio between carotenoids and chlorophylls. Table 1 shows the results obtained for the 15 runs, whereas the estimated response surfaces for the different response variables are shown in Figure 3. Additionally, in order to remark the significance of the experimental factors on the response variables, the standardized Pareto charts for these variables are included in Figure S3.

As can be observed, the extraction yield varied significantly depending on the extraction conditions (from 20.6 to $51.9 \%$ ), reaching the highest value when the temperature was the highest $\left(120^{\circ} \mathrm{C}\right)$. Furthermore, the composition of the extracting solvent seemed to have a relevant influence on this factor, as it can be also observed in Figure $3 \mathrm{~A}$. Moreover, the extraction yield increased positively with the percentage of ethanol in the solvent mixture and with the number of extraction cycles. In contrast, carotenoid content seemed to behave the opposite way. As Figure 3B shows, the percentage of ethanol in the solvent mixture exhibited a negative and significant 
effect on the total carotenoid content, along with the temperature. This effect was also observed by Sanchez-Camargo et al. [25] during the extraction of fucoxanthin from $P$. tricornutum by PLE using ethanol or ethyl acetate. In this sense, a higher extraction yield was also observed using ethanol, while a higher carotenoids content was reached using ethyl acetate. Furthermore, in this case, the number of cycles did not seem to affect significantly to carotenoid content in the extract although fewer number of cycles was preferred. This fact may indicate that carotenoids could have been fully extracted in one cycle and no more solvent is necessary to extract these components from the matrix. Finally, the ratio between carotenoids and chlorophylls was significantly affected by extraction temperature (Figure 3C), thus lower temperatures were preferred.

Keeping in mind that total carotenoids content was the most important parameter to be maximized, the optimization of PLE process was thus only focused on the total carotenoids response, and the optimum values of the experimental factors were selected considering these results. Thus, the optimization of the PLE procedure to obtain the highest possible amounts of carotenoids in the extracts indicated that the use of 100 $\%$ ethyl acetate at $40{ }^{\circ} \mathrm{C}$ and one static extraction cycle were more favorable. Under these conditions, the experimental result obtained for carotenoids content was 132.81

$371 \pm 3.05 \mathrm{mg}$ per $\mathrm{g}$ extract, which was very similar to the value predicted by the model 372 (142.77 mg per g extract).

\subsection{In-line purification of fucoxanthin during pressurized liquid extraction}

374 The optimum extract obtained in the previous section was rich in carotenoids, mainly fucoxanthin, but it also contained significant amounts of chlorophylls (Table 1),

376 specifically pheophytin $a$, which is a chlorophyll a-type compound. Chlorophylls are often co-extracted with carotenoids, considering their relatively similar polarities, 
378 although they are not so appreciated in the final products in which carotenoids are

379 added. For this reason, it is not only interesting to extract as many carotenoids as

380 possible from the natural sources but also to obtain extracts as pure as possible, with

381 less interfering compounds. PLE is able to produce high extraction yields and fast

382 processes but this technique is also less selective towards specific components than

383 others, such as supercritical fluid extraction [10]. In this sense, the design of approaches

384 for the in-line purification of carotenoids extracted by PLE is very interesting. A viable alternative is the use of adsorbents that are able to selectively retain some of the extracted compounds. In the present work, different adsorbents were tested with the aim to produce selective retention of the extracted chlorophylls, thus, generating at the same time more purified extracts on carotenoids. All the extractions were performed as described in the experimental section, including $1 \mathrm{~g}$ of adsorbent in the extraction cell (Figure 1B) and selecting the most appropriate PLE conditions previously optimized.

Table 2 shows the results obtained with 5 different adsorbents. Carotenoids and chlorophylls content on the resulting extracts as well as their ratio are included. charcoal, with 14.5 and $5.5 \%$ respectively, showed a lower value in comparison with the extract that was obtained without adsorbent. Interestingly, most adsorbents, except for aluminum oxide, produced retention of carotenoids to some extent, illustrated by the statistically significantly lower values of total carotenoids in the resulting extracts. When the content of chlorophylls was analyzed, a huge decrease of extracted chlorophylls was observed using activated charcoal and, although total carotenoid content was also very low, the ratio between these two groups of pigments was significantly higher (Table 2). Moreover, as can be observed in Figure S4, the extract obtained with activated charcoal 
showed an orange-brown color that apparently indicated the absence of chlorophylls in the extract. This fact was confirmed by the HPLC analysis shown in Figure 4. As mentioned, fucoxanthin (peak 1 , see Table 3 ) was the main carotenoid present in T. lutea PLE extracts, although other compounds such as diadinoxanthin (peak 4), diatoxanthin were also present. Regarding chlorophylls, pheophytin a (peak 10) was detected in the optimum PLE extract. The extracts produced using adsorbents presented a similar HPLC profile (data not shown), excepting the one obtained with activated charcoal. As can be clearly appreciated in Figure 4, activated charcoal produced the retention of all the chlorophyll-related components. Indeed, the only peak present was identified as fucoxanthin, although its concentration was lower compared to the rest of the extracts. Considering these promising results, an optimization of the use of activated charcoal as an adsorbent during PLE was carried out.

\subsection{In-line purification of fucoxanthin during pressurized liquid extraction using} activated charcoal

As it was confirmed in the previous section, activated charcoal seemed to remove the chlorophylls from the extract, although carotenoids were also partially retained. To more closely study the behavior of the adsorbent during the PLE process, six different amounts of activated charcoal ( 0.25 to $1.0 \mathrm{~g} \mathrm{AC}$ ) were tested during PLE extractions with the aim to maximize the retention of chlorophylls while maximizing the recovery of fucoxanthin. Results obtained can be observed in Figure 5 whereas the obtained extracts are pictured in Figure S5. Independently of the extraction conditions tested, when activated charcoal was added into the extraction cell, a decrease of both types of pigments, carotenoids, and chlorophylls, occurred. However, chlorophylls' retention 
426 had a more marked trend as the amount of adsorbent was increased. In fact, when 0.6

427 g of activated charcoal were used, the amount of chlorophylls recovered in the extracts

428 was extremely low, while the amount of fucoxanthin recovered was still high. Above 0.6

$429 \mathrm{~g}$, carotenoids were strongly retained and, thus, those extraction and purification

430 conditions were not considered suitable. In agreement with those results (Figure 5),

431 placing a layer of $0.6 \mathrm{~g}$ of activated charcoal at the exit of the extraction cell (Figure 1B)

432 allowed reaching total carotenoids and total chlorophylls contents of $97.64 \mathrm{mg}$ per $\mathrm{g}$

433 extract and $0.42 \mathrm{mg}$ per g extract, respectively. As a result, the extracts obtained under

434 those simultaneous extraction and purification conditions presented a carotenoids-to-

435 chlorophylls ratio of 252.51 which was 80 -fold higher than the one present in the original

436 PLE extract without adsorbent. As a further confirmation of those observations, the

437 HPLC analysis of the obtained extracts using different amounts of activated charcoal

438 confirmed the absence of chlorophylls when $0.6 \mathrm{~g}$ of activated charcoal were used

439 (Figure S6).

440

441

\section{CONCLUDING REMARKS}

442 A new process for the simultaneous pressurized extraction and purification of carotenoids has been developed as an environmentally green method to obtain a chlorophyll-free and fucoxanthin-enriched extract from Tisochrysis lutea microalga. Firstly, a rapid screening of two different batches of $T$. lutea biomass indicated that these microalgae increased their content in fucoxanthin when natural light was used in opposite to LED irradiation supplementation during cultivation. Then, an experimental design was applied to determine the most suitable PLE conditions to obtain a carotenoid-rich extract from $T$. lutea. The use of pressurized ethyl acetate at $40^{\circ} \mathrm{C}$ using 

extract containing $132.81 \mathrm{mg}$ carotenoids per g of extract. Subsequently, this PLE 452 process was further improved to produce an in-line purification of carotenoids using activated charcoal as adsorbent ( $0.6 \mathrm{~g}$ per extraction). The use of activated charcoal at

454 those conditions permitted to selectively retain the chlorophylls present in the extracts 455 efficiently allowing the attainment of purified carotenoids-enriched extracts from $T$. $456 \quad$ lutea biomass.

459 Authors would like to thank projects ABACUS (Algae for a Biomass Applied to the production of added value compoundS, grant agreement No 745668, funded by the Bio Based Industries Joint Undertaking under the European Union's Horizon 2020 research and innovation programme) and AGL2017-89417-R (MINECO, Spain) for financial support. Authors also thank A4F - Algae for Future, Portugal, for supplying and assisting with algae biomass.

465

\section{CONFLICT OF INTEREST STATEMENT}

467 The authors would like to declare no conflicts of interest in the publication of this 468 research. 
472 [1] Gallego R., Montero L., Cifuentes A., Ibáñez E., Herrero M., Green Extraction of 473 Bioactive Compounds from Microalgae. J. Anal. Test. 2018, 2, 109-123.

474 [2] Guedes A. C., Amaro, H. M. Malcata, F. X., Microalgae as sources of carotenoids. Mar. Drugs 2011, 9, 625-644.

476

[3] Mulders K. J. M., Weesepoel Y., Lamers P. P., Vincken J. P., Martens D. E., Wijffels galbana T-ISO. J. Appl. Phycol. 2013, 25, 1421-1430.

[4] Bendif E. M., Probert I., Schroeder D. C., de Vargas C., On the description of 480

[5] Bigagli E., Cinci L., Niccolai A., Biondi N., Rodolfi L., D’Ottavio M., D’Ambrosio M., Lodovici M., Tredici M. R., Luceri C., Preliminary data on the dietary safety, tolerability and effects on lipid metabolism of the marine microalga Tisochrysis lutea. Algal Res. 2018, 34, 244-249.

[6] Rodriguez-Concepcion M., Avalos J., Bonet M. L., Boronat A., Gomez-Gomez L., Hornero-Mendez D., Limon M. C., Meléndez-Martínez A. J., Olmedilla-Alonso B., Palou A., Ribot J., Rodrigo M. J., Zacarias L., Zhu C., A global perspective on carotenoids: Metabolism, biotechnology, and benefits for nutrition and health. Prog. Lipid Res. 2018, 70, 62-93.

[7] Matos J., Cardoso C., Bandarra N. M., Afonso C., Microalgae as healthy ingredients for functional food: A review. Food Funct. 2017, 8, 2672-2685. 
[8] Kim S. M., Kang S. W., Kwon O. N., Chung D., Pan C. H., Fucoxanthin as a major carotenoid in Isochrysis aff. galbana: Characterization of extraction for commercial application. J. Korean Soc. Appl. Biol. Chem. 2012, 55, 477-483.

[9] Poojary M. M., Barba F. J., Aliakbarian B., Donsì F., Pataro G., Dias D. A., Juliano P., Innovative alternative technologies to extract carotenoids from microalgae and seaweeds. Mar. Drugs 2016, 14 (11), 214.

[10] Gallego R., Bueno M., Herrero M., Sub- and supercritical fluid extraction of bioactive compounds from plants, food-by-products, seaweeds and microalgae An update. TrAC - Trends Anal. Chem. 2019, 116, 198-216.

[11] Islam A. K. M. M., Lee H. S., Ro J. H, Kim D., Kwon H. Application of high-surfacearea graphitized carbon black with primary secondary amine as an alternative quick, easy, cheap, effective, rugged, and safe clean-up material for pesticide multiresidue analysis in spinach. J. Sep. Sci. 2019, 42, 2379-2389.

[12] Cabrera L. C, Caldas S. S., Prestes O. S., Primel E. G. Zanella R. Evaluation of alternative sorbents for dispersive solid-phase extraction clean-up in the QuEChERS method for the determination of pesticide residues in rice by liquid chromatography with tandem mass spectrometry. J. Sep. Sci. 2016, 39, 1945-1954.

[13] Han L., Sapozhnikova Y., Matarrita J. Evaluation of a new carbon/zirconia-based sorbent for the clean-up of food extracts in multiclass analysis of pesticides and environmental contaminants. J. Sep. Sci. 2016, 39, 4592-4602

[14] Osman R., Saim N., Selective extraction of organic contaminants from soil using pressurised liquid extraction. J. Chem. 2013, ID 357252. 
[15] Fujii K., Process integration of supercritical carbon dioxide extraction and acid treatment for astaxanthin extraction from a vegetative microalga. Food Bioprod. Process. 2012, 90, 762-766.

[16] Li T., Xu J., Wu H., Wang G., Dai S., Fan J., He H., Xiang W., A saponification method for chlorophyll removal from microalgae biomass as oil feedstock. Mar. Drugs 2016, 14, 162.

[17] Gilbert-López B., Mendiola J. A., van den Broek L. A. M., Houweling-Tan B., Sijtsma L., Cifuentes A., Herrero M., Ibáñez E., Green compressed fluid technologies for downstream processing of Scenedesmus obliquus in a biorefinery approach. Algal Res. 2017, 24, 111-121.

[18] Gilbert-López B., Mendiola J. A., Fontecha J., Van Den Broek L. A. M., Sijtsma L., Cifuentes A., Herrero M., Ibáñez E., Downstream processing of Isochrysis galbana: a step towards microalgal biorefinery. Green Chem. 2015, 17, 4599-4609.

[19] Gallego R., Martínez M., Cifuentes A., Ibáñez E., Herrero M., Development of a Green Downstream Process for the Valorization of Porphyridium cruentum Biomass. Molecules 2019, 24, 1564.

[20] Jin E., Polle J. E. W., Lee H. K., Hyun S. M., Chang Man, Xanthophylls in microalgae: from biosynthesis to biotechnological mass production and application. Microb Biotechnol. 2003, 13, 165-174.

[21] Xu R., Gong Y., Cheng W., Li S., Chen R., Zheng X., Cheng X., Wang H., Effects of LED monochromatic light quality of different colors on fucoxanthin content and expression levels of related genes in Phaeodactylum tricornutum. Acta Opt. Sin. 2019, 39, 0917001. 
[22] Reyes F. A., Mendiola J. A., Ibañez E., Del Valle J. M., Astaxanthin extraction from Haematococcus pluvialis using $\mathrm{CO}_{2}$-expanded ethanol. J. Supercrit. Fluids 2014, 92, 75-83.

[23] Castro-Puyana M., Herrero M., Urreta I., Mendiola J. A., Cifuentes A., Ibáñez E., Suárez-Alvarez S., Optimization of clean extraction methods to isolate carotenoids from the microalga Neochloris oleoabundans and subsequent chemical characterization using liquid chromatography tandem mass spectrometry. Anal. Bioanal. Chem. 2013, 405, 4607-4616.

[24] Gilbert-López B., Barranco A., Herrero M., Cifuentes A., Ibáñez E., Development of new green processes for the recovery of bioactives from Phaeodactylum tricornutum. Food Res. Int. 2017, 99, 1056-1065.

[25] del Pilar Sánchez-Camargo A., Pleite N., Herrero M., Cifuentes A., Ibáñez E., Gilbert-López B., New approaches for the selective extraction of bioactive compounds employing bio-based solvents and pressurized green processes. J. Supercrit. Fluids 2017, 128, 112-120.

[26] McClure D. D., Luiz A., Gerber B., Barton G. W., Kavanagh J. M., An investigation into the effect of culture conditions on fucoxanthin production using the marine microalgae Phaeodactylum tricornutum. Algal Res. 2018, 29, 41-48.

[27] Shang Y. F., Kim S. M., Lee W. J., Um B.-H., Pressurized liquid method for fucoxanthin extraction from Eisenia bicyclis (Kjellman) Setchell. J. Biosci. Bioeng. $2011,111,237-41$.

[28] Kim S. M., Jung Y. J., Kwon O. N., Cha K. H., Um B. H., Chung D., Pan C. H., A potential commercial source of fucoxanthin extracted from the microalga Phaeodactylum tricornutum. Appl. Biochem. Biotechnol. 2012, 166, 1843-1855. 
565 Figure 1. Packing of the PLE extraction cell. (A) Without adsorbent; (B) with adsorbent.

566 Figure 2. Cultivation of $T$. lutea in a flat-panel photobioreactor of $270 \mathrm{~L}$ in Lisbon from

567 August to November with natural light (NL, A) or with double-sided LEDs illumination 568 (LED, B).

569 Figure 3. Estimated response surface obtained for extraction yield (A), total carotenoids content (B) and ratio between carotenoids and chlorophylls (C) for the optimization of

571 carotenoids in T. lutea PLE extracts.

572 Figure 4. Chromatogram $(450 \mathrm{~nm})$ obtained by HPLC-DAD from the optimum Tisochrysis 573 lutea PLE extract (in green) and the same using activated charcoal as adsorbent (in 574 orange). For peak assignment, see Table 3.

575 Figure 5. Total carotenoids and chlorophylls contents of extracts using different 576 amounts of activated charcoal (AC) during PLE of T. lutea. 
578 Table 1. Experimental design including factors and values of the response variables 579 studied for the PLE optimization. CP: central points

\begin{tabular}{|c|c|c|c|c|c|c|}
\hline $\begin{array}{c}\text { Exp. } \\
\text { \# }\end{array}$ & $\begin{array}{l}\% \mathrm{EtOH} \\
\text { in EtAc }\end{array}$ & $\begin{array}{c}\mathrm{T} \\
\left({ }^{\circ} \mathrm{C}\right)\end{array}$ & Cycle & $\begin{array}{c}\text { Yield } \\
\text { (\%) }\end{array}$ & $\begin{array}{l}\text { Carotenoids } \\
\text { (mg per g } \\
\text { extract }_{D W} \text { ) }\end{array}$ & Ratio car/chl \\
\hline 1 & 0 & 40 & 2 & $20.6 \pm 0.3$ & $128.80 \pm 0.34$ & $2.95 \pm 0.04$ \\
\hline 2 & 0 & 80 & 3 & $23.0 \pm 1.5$ & $121.43 \pm 4.43$ & $2.21 \pm 0.10$ \\
\hline 3 & 0 & 80 & 1 & $24.7 \pm 2.6$ & $121.39 \pm 3.09$ & $2.26 \pm 0.01$ \\
\hline 4 & 0 & 120 & 2 & $27.7 \pm 1.1$ & $99.49 \pm 1.39$ & $2.04 \pm 0.05$ \\
\hline 5 & 50 & 40 & 3 & $35.2 \pm 1.9$ & $98.61 \pm 9.49$ & $2.63 \pm 0.53$ \\
\hline 6 & 50 & 40 & 1 & $28.8 \pm 1.7$ & $110.06 \pm 0.43$ & $2.58 \pm 0.26$ \\
\hline $7-9$ (CP) & 50 & 80 & 2 & $40.4 \pm 2.3$ & $76.69 \pm 5.59$ & $2.58 \pm 0.65$ \\
\hline 10 & 50 & 120 & 3 & $51.9 \pm 0.3$ & $54.72 \pm 7.72$ & $1.91 \pm 0.20$ \\
\hline 11 & 50 & 120 & 1 & $45.8 \pm 1.1$ & $56.86 \pm 10.26$ & $1.88 \pm 0.15$ \\
\hline 12 & 100 & 40 & 2 & $32.0 \pm 0.7$ & $86.64 \pm 4.60$ & $2.70 \pm 0.48$ \\
\hline 13 & 100 & 80 & 3 & $39.0 \pm 1.0$ & $66.48 \pm 8.82$ & $2.93 \pm 0.16$ \\
\hline 14 & 100 & 80 & 1 & $35.2 \pm 3.3$ & $72.06 \pm 7.16$ & $2.93 \pm 0.67$ \\
\hline 15 & 100 & 120 & 2 & $44.0 \pm 5.4$ & $60.72 \pm 8.63$ & $2.07 \pm 0.36$ \\
\hline
\end{tabular}

580 
Table 2. Effect of different adsorbents for in-cell purification of carotenoids during PLE

583 at optimum conditions. Different superscript letters indicate statistically significant 584 differences $(p<0.05)$.

\begin{tabular}{|c|c|c|c|c|}
\hline Adsorbent & $\begin{array}{c}\text { Yield } \\
(\%)\end{array}$ & $\begin{array}{l}\text { Carotenoids } \\
\text { (mg per g } \\
\text { extract }_{D w} \text { ) }\end{array}$ & $\begin{array}{l}\text { Chlorophylls } \\
\text { (mg per g } \\
\text { extract }_{\mathrm{DW}} \text { ) }\end{array}$ & Ratio car/chl \\
\hline None & $18.3 \pm 0.7^{a}$ & $132.81 \pm 3.05^{b}$ & $42.47 \pm 0.91^{c}$ & $3.13 \pm 0.14^{b}$ \\
\hline Isolute C-18 & $18.4 \pm 0.5^{a}$ & $116.70 \pm 3.81^{c}$ & $44.84 \pm 1.51 \mathrm{bc}$ & $2.60 \pm 0.00^{b}$ \\
\hline Isolute ENV+ & $17.7 \pm 0.2^{a}$ & $110.50 \pm 3.41^{c}$ & $46.25 \pm 0.72^{b}$ & $2.39 \pm 0.04^{b}$ \\
\hline Aluminum oxide & $14.5 \pm 0.9^{b}$ & $154.10 \pm 112.15^{a}$ & $61.63 \pm 2.01^{a}$ & $2.50 \pm 0.15^{b}$ \\
\hline Molecular sieve & $19.3 \pm 0.7^{\mathrm{a}}$ & $112.15 \pm 1.40^{c}$ & $47.42 \pm 1.69^{b}$ & $2.37 \pm 0.11^{b}$ \\
\hline Activated charcoal & $5.5 \pm 1.1^{c}$ & $31.60 \pm 8.11^{d}$ & $0.23 \pm 0.12^{d}$ & $154.69 \pm 62.16^{a}$ \\
\hline
\end{tabular}

585

586

587

588 
590 of the compounds in the T. lutea PLE extracts.

\begin{tabular}{|c|c|c|c|c|}
\hline $\begin{array}{l}\text { Peak } \\
\#\end{array}$ & $\begin{array}{l}t_{R} \\
(\min )\end{array}$ & Identification & $\begin{array}{l}\text { UV-Vis } \\
\text { maxima (nm) }\end{array}$ & $\begin{array}{l}{[\mathrm{M}+\mathrm{H}]^{+} \mathrm{m} / \mathrm{z}} \\
\text { and main } \\
\text { fragments }\end{array}$ \\
\hline 1 & 6.969 & All-trans fucoxanthin ${ }^{*}$ & $420 s, 445,472$ & 641 \\
\hline 2 & 7.435 & cis-fucoxanthin & $420 s, 442,465$ & 659,641 \\
\hline 3 & 7.745 & cis-fucoxanthin & $420 s, 438,458$ & 659,641 \\
\hline 4 & 14.973 & All-trans diadinoxanthin & $420 s, 444,474$ & 583 \\
\hline 5 & 16.959 & Carotenoid type & $405 s, 429,456$ & $749,663,584$ \\
\hline 6 & 17.804 & Carotenoid type & $405 s, 429,456$ & 584,569 \\
\hline 7 & 20.017 & All-trans diatoxanthin & $424 s, 450,478$ & 567 \\
\hline 8 & 22.530 & Fucoxanthin derivative & $420 s, 458,476$ & - \\
\hline 9 & 24.261 & Fucoxanthin derivative & $420 s, 458,478$ & - \\
\hline 10 & 25.737 & Pheophythin $a$ & $373 s, 408,666$ & 872,593 \\
\hline 11 & 26.143 & $\beta$-carotene ${ }^{*}$ & $424 s, 450,478$ & 537 \\
\hline
\end{tabular}

591 




593

594 Figure 1

595 


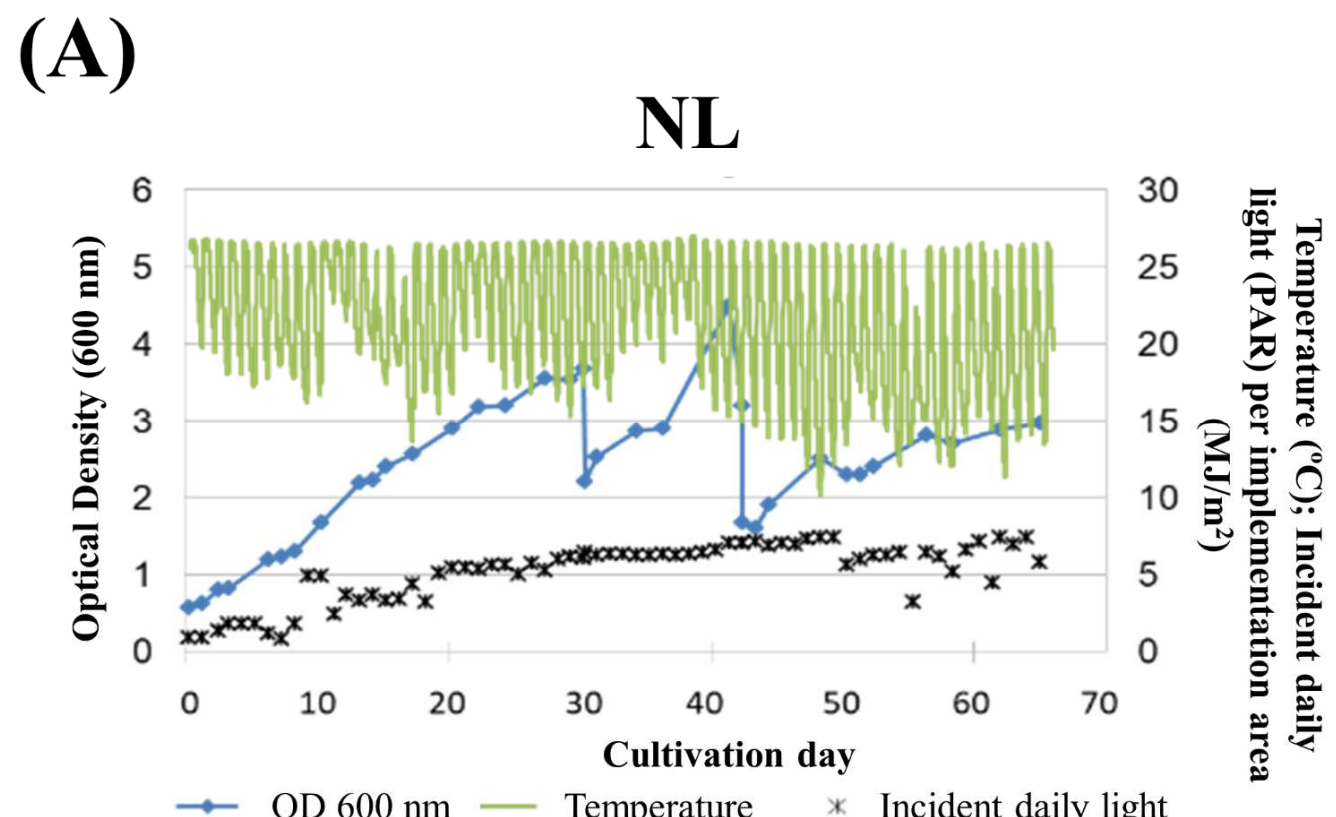

(B)



Figure 2 
(A) Extraction yield

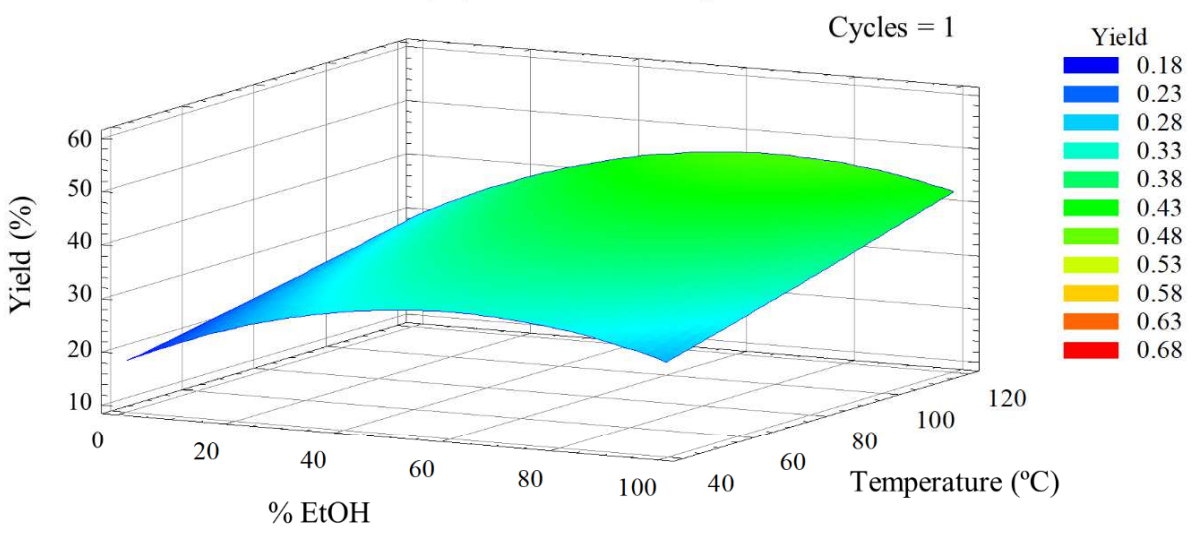

(B) Total carotenoids



(C) Ratio carotenoids/chlorophylls

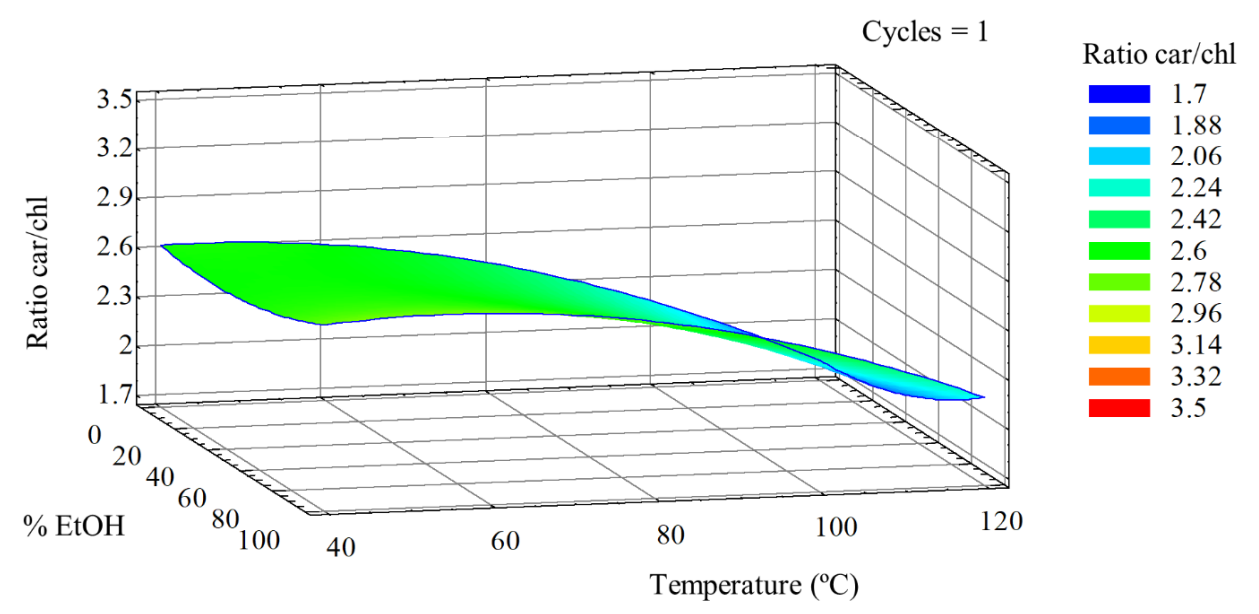

Figure 3 


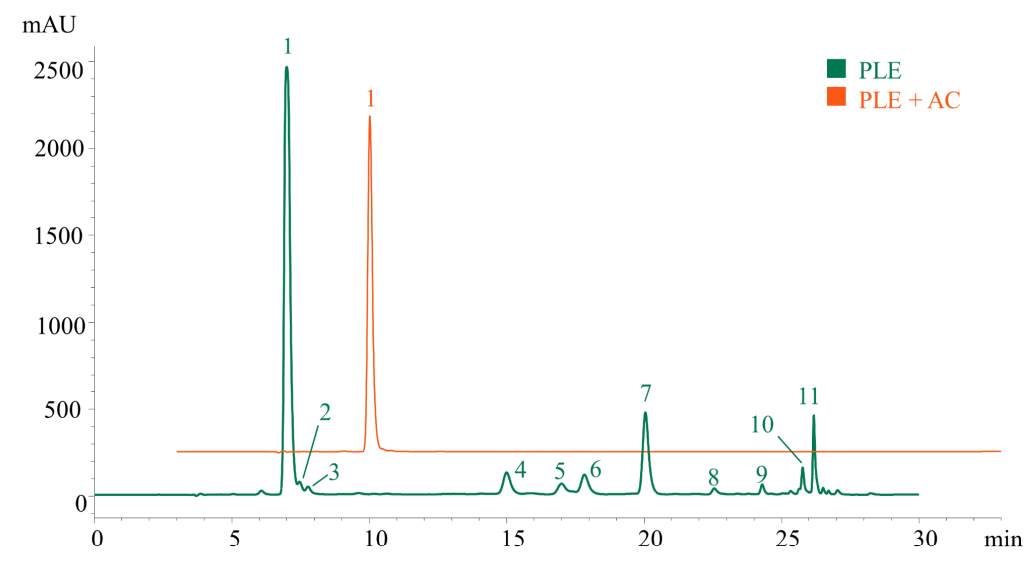

602

$603 \quad$ Figure 4

604 


\section{Carotenoids and chlorophylls content}

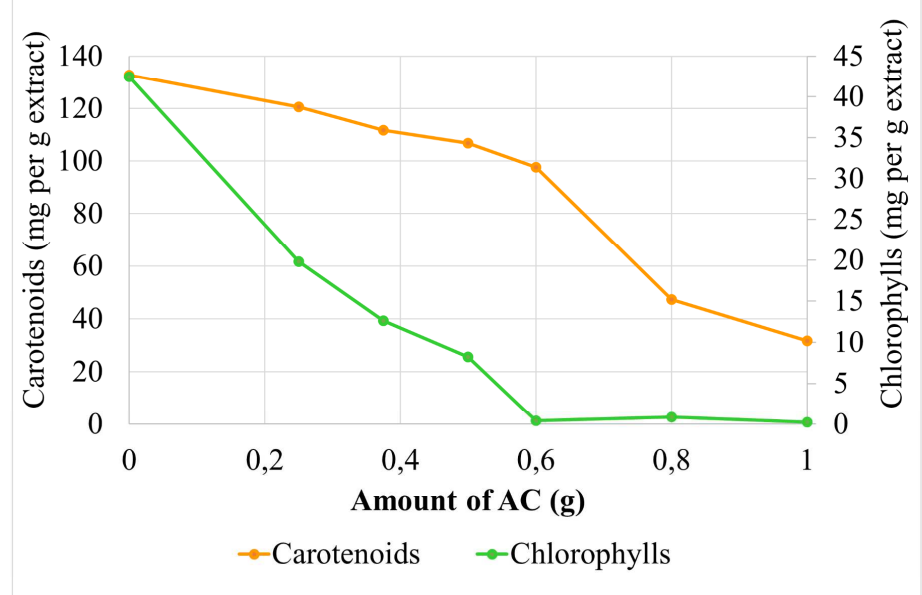

605

606 Figure 5 